\title{
Concurrent cognitive task may improve motor work performance and reduce muscle fatigue
}

\author{
Maria Evstigneeva ${ }^{\mathrm{a}}$, Aleksandr Aleksandrov ${ }^{\mathrm{a}}$, Svend Erik Mathiassen ${ }^{\mathrm{b}}$ and Eugene Lyskov ${ }^{\mathrm{b}}$ \\ ${ }^{a}$ Saint Petersburg State University, Saint Petersburg, Russia \\ ${ }^{\mathrm{b}}$ Gävle University, Gävle, Sweden
}

\begin{abstract}
Performance of certain cognitive tasks either during physical load or in rest pauses between boosts might lead to slowing of muscle fatigue and fatigue related decline in performance. Seventeen right-handed healthy volunteers (age $24 \pm 1.4$, 8 males) participated in this study, aiming to investigate the effect of the level of the cognitive information processing - 1) passive perception of audio stimuli, 2) active stimuli discrimination, 3) active stimuli discrimination following motor response - on motor task performance (handgrip test $30 \%$ and $7 \%$ of MVC) and muscle fatigue development. Cognitive tasks show the following effects on motor work: i) Perceived fatigue during $30 \% \mathrm{MVC}$ (fatiguing) condition developed slower if participant pressed button in response to deviant acoustic stimuli, as compared to passive listening. Counting task, an active task without motor component, took the intermediate position and did not differ significantly from two other cognitive tasks. ii) MVC after $30 \%$ MVC (fatiguing) condition tended to decrease stronger when accompanied with passive listening in comparison with both active tasks. iii) Motor task performance during 30\% MVC (fatiguing) condition was better for active cognitive task with motor component than for passive task. Active task without motor component took the intermediate position and did not differ significantly from both the other cognitive tasks.
\end{abstract}

Keywords: Handgrip test, dual task paradigm, cognitive information processing, central fatigue

\section{Introduction}

Motor work leads to muscle fatigue, which is a decline in a person's ability to exert force (1) including both an increase in the perceived effort necessary to exert the desired force and an eventual inability to produce such force (2). Thus muscle fatigue is thought to originate not only from the peripheral component, caused by the metabolic changes in muscles but also from the central effects.

It was shown that execution of cognitive task influence on motor performance and development of muscular fatigue. For example, muscle fatigue occurs slower if the participants perform rather difficult memory tasks during pauses in muscle load. Diversity and variation of loads under certain favourable proportions can significantly increase endurance and enhance relief of activated muscle fibers (3). Certain cognitive tasks simultaneously with physical load or during short rest between work boosts may increased resistance to fatigue and accelerate recuperation.

Stride width variability - an index of a locomotor control - also appears to be sensitive to the level of cognitive demands imposed by the dichotic listening condition, constituting the concurrent task (4). Better knowledge about interaction between motor and cognitive processes seems to be important to optimize labour management, to improve preventive measures of fatigue development and to increase labour productivity.

The aim of this experimental study was to investigate the effects of the level of the cognitive engagement -1 ) passive perception of audio stimuli, 2) active stimuli discrimination, 3 ) active stimuli discrimination following motor response - on concurrent motor task performance and muscle fatigue development. We test hypothesis that certain stages of cognitive task performance would slow down devel-

\footnotetext{
* Corresponding author. Email: maria.evstigneeva@gmail.com
} 
opment of perceived fatigue and fatigue-related decline in motor performance in comparison with passive listening. Handgrip test with $30 \%$ of MVC (fatiguing) and $7 \%$ of MVC (control) was applied as a motor task

\section{Materials and Methods}

Seventeen right-handed undergraduate, graduate and $\mathrm{PhD}$ students of St. Petersburg State University (age $24 \pm 1.4,8$ males); took part in the experiment. All reported normal hearing and gave their informed consent having the testing procedure explained to them.

The experiment had a $2 \times 3$ crossover design produced by the combination of two force production levels and three cognitive tasks. Only the effects of concurrent cognitive task on motor task performance are discussed in this paper.

The motor load consisted in sustaining a handgrip of dynamometer with the right-hand at a target force level $(30 \%$ of participant's maximal voluntary contraction (MVC) - highly fatiguing muscular load, and $7 \%$ MVC - light work) during four sequential oddball-blocks (2.7 minutes each, with $30 \mathrm{~s}$. intervals) of dual-task (Load) period. Sustained contraction at force levels above $25 \%$ of the maximal force is known to result in fatigue. Participants saw target and actually produced force levels as two lines of different colors at the monitor.

Cognitive tasks included: passive listening of acoustic stimuli in the oddball paradigm (participants were watching silent movie and had no task related to the acoustic stimuli - a sequence of frequent "standards" sometimes interspersed with rare "deviants"; passive task), counting of the rare stimuli of the same sequence (active task without motor component), and button press with the participant's left hand in response to those rare stimuli, as quick as possible (active task with motor component).

The stimuli were two computer generated sinusoidal tones of $60 \mathrm{~dB}$ SPL, $100 \mathrm{~ms}$ duration, 10 ms rise and fall times, and $1000 \mathrm{~Hz}$ (frequent stimulus, standard) and $1032 \mathrm{~Hz}$ (rare stimulus, deviant, target in active tasks) pitch. Stimuli were presented to the participants with $800 \mathrm{~ms}$ onset asynchronies (SOA) in four sequential oddballparadigm blocks of 200 sounds each with 30 s. interblock intervals during each of the periods before (PreLoad), during (Load, dual task period), and after (PostLoad) muscular work performance.
Procedure. Participants were comfortably seated in a chair and instructed to relax as far as possible, keep their eyes open, and to avoid unnecessary movements. Auditory stimuli were delivered binaurally via headphones. Participants were watching a silent movie during the periods without motor load. Computer monitor for force level monitoring (dual task period) and movie showing (periods of the passive task without muscular work) was situated at approximately 0.5 $\mathrm{m}$ from subject's eyes; dynamometer was under the right and the keyboard (for button press) was under the left hand. Force level was monitored using a USB-connected A/D converter; National Instruments Data Acquisition Device "NI USB-6009" (14 bit resolution, sampled at $100 \mathrm{~Hz}$ ) and LabView v. 8.2.1 custom scripts. The motor task was declared to be the priority one.

The three cognitive tasks were performed by every participant in three different days counterbalanced across the participants. The $30 \% \mathrm{MVC}$ and $7 \%$ MVC sessions for each cognitive task were performed in the same day in a 20-30 min pause for rest. The order of the force-level sessions performance was constant during all the cognitive tasks for individual participant and counterbalanced across all the participants.

Recordings

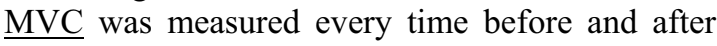
motor load as a maximal voluntary handgrip, averaged for $5 \mathrm{~s}$ contracting.

Motor task performance was estimated visually according to 5-degree scale (100\% of work performed obtained 5 , the grade decreased by one point for every $20 \%$ drop).

Perceived muscle fatigue was evaluated according to Borg's CR-10 scale (Borg, 1998) before the task start, after each block termination during the motor task performance, and at the end of experimental session.

Cognitive task performance. The number of counted deviant stimuli was reported by the participant verbally after each block. Button presses were registered using Presentation 11.0 (www.neurobs.com), which also presented the auditory stimuli.

Statistical analyses were performed with SPSS 11.5 (SPSS Inc., Chicago, IL, USA). Analysis of variance (ANOVA) with repeated measures and Bonferroni-corrected post hoc multiple comparison procedure was used to examine the effects of time, cognitive task, and force level. The Greenhouse-Geisser correction was applied when a Mauchly's test showed sphericity violation; in those cases epsilon 
(e) is shown. Data on perceived fatigue from one women-participant in button press session were not included in the analysis because, as she said later, she

\section{Results}

MVC decreased significantly after the $30 \% \mathrm{MVC}$ contraction protocol $(\mathrm{F}(1)=30.17 \mathrm{p}<0.001)$, did not change significantly after the $7 \%$ MVC contraction protocol $(\mathrm{p}>0.5)$, and was significantly lower after the $30 \%$ than after $7 \%$ contraction protocol $(F(1)=80.12 \mathrm{p}<0.001)$, though there was no difference between force level conditions before the motor task start $(\mathrm{p}>0.7)$. No significant effects of or interactions with Cognitive task were found, however MVC tended to decrease more after $30 \%$ MVC contraction protocol combined with passive cognitive task, as compared to the other two tasks.
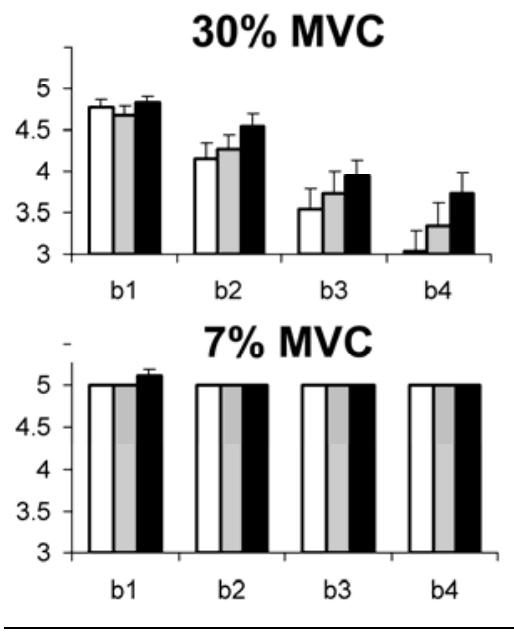

Figure 1. Decrease in performance (scores) during $30 \%$ and 7\% MVC protocols

Motor task performance All the participants succeeded in performing the $7 \%$ MVC contraction protocol at the target force level during all the four blocks of the Load period. For $30 \%$ MVC contraction protocol motor task performance decreased significantly from the first block to the fourth one (Fig 1.) $(\mathrm{F}(3)=44.85 \mathrm{e}=0.55 \mathrm{p}<0.001$, Post Hoc test: all the blocks differed from each other, $\mathrm{p} \leq 0.003)$. During the highly fatiguing muscle load there was a marginally significant tendency for better performance in the button press cognitive task $(F(2)=2.77 \mathrm{p}=0.078)$. That tendency became significant by the fourth block could not estimate her fatigue adequately due to hard emotional exertion she met that day.1).

$(F(2)=3.79 \mathrm{e}=0.732 \mathrm{p}=0.049)$, where motor performance was better for button press condition then for the passive one (Post Hoc test: $p=0.043$ ). Counting task occupied the intermediate position and did not differ significantly from the both other cognitive tasks.

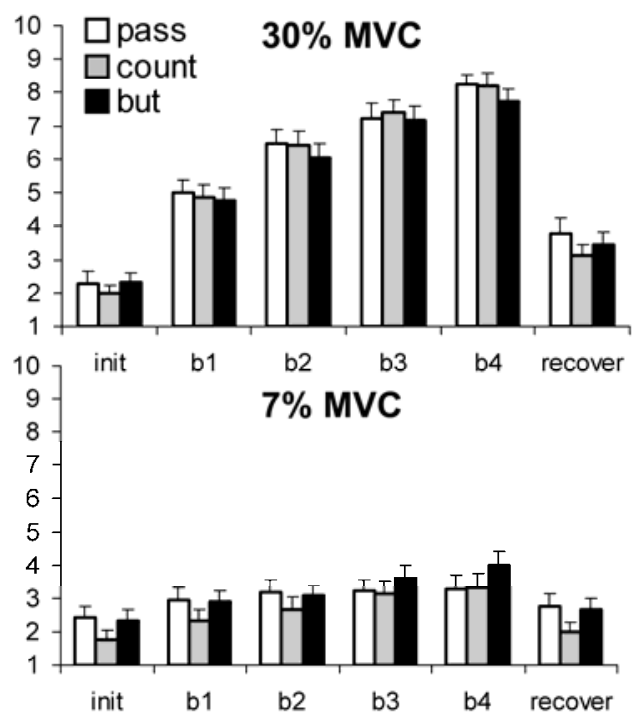

Figure 2. Percieved fatigue (Borg's CR 10 scale) during $30 \%$ and $7 \%$ MVC protocols

Perceived fatigue in overall ANOVA demonstrated significant dependence on Force $(F(1)=115.18$ $\mathrm{p}<0.001)$, Time $(\mathrm{F}(5)=53.67 \mathrm{e}=0.277 \mathrm{p}<0.001)$, and the interaction Force and Time $(\mathrm{F}(1,5)=55.43$ $\mathrm{e}=0.548 \mathrm{p}<0.001$ ), and of Force, Time and Cognitive task $(F(1,5,2)=1.91 \mathrm{p}=0.048)$. There was no significant effects for the initial time point (before the tasks start), but for each block of the Load period $(p<0.001)$ and after recovery (at the end of the experimental session, $p=0.007$ ) perceived fatigue was higher for the $30 \%$ MVC contraction protocol than for the $7 \%$ one. (Figure 2). Furthermore, for the last block of the Load period there was a significant interaction of Force and Cognitive task $(\mathrm{F}(1,2)=6.83$ $\mathrm{p}=0.004)$. Detailed analysis revealed significant effect of Cognitive task for the fourth block during $30 \%$ MVC contraction protocol performance $(\mathrm{F}(2)=$ $4.22 \mathrm{p}=0.025)$ : when the participants were to press the button in response to the deviant, perceived fatigue was significantly lower, than during passive lis- 
tening of the stimuli (Post Hoc test, $\mathrm{p}=0.018$ ). Counting task did not differ significantly from both the other cognitive tasks.

\section{Discussion}

Data obtained in this study show that motor component of cognitive, senso-motor task (button press) associates with slowing in development of perceived muscle fatigue and fatigue related motor performance.

MVC, as expected, decreased after $30 \%$, MVC but not after $7 \%$ MVC workload. Motor performance decreased during $30 \%$ workload but was stable at target level during $7 \%$ load. Perceived fatigue in $30 \%$ workload condition was increased during and after motor work. It grew with time during the motor work execution, and was higher during and after $30 \%$ than $7 \%$ workload. Thus, motor work execution at $30 \%$ MVC force level indeed resulted in distinctive fatigue.

The effect of presence of motor component in cognitive task on the motor exercise performance and fatigue development agrees with ideas expressed by Sechenov I.M. as far back as the 19-th century as well as with more recent data on the facilitative effect of motor exercise on the motor part of cognitive task performance $(5,6)$ Sechenov described the phenomenon of faster recovery of fatigued muscle after load in case of activity change, as compared to complete rest. This effect was explained in terms of "simultaneous negative induction" - an emergence of a new excitation center, caused by activation of a new muscle, intensifies the inhibition processes in a cortical representation of fatigued muscle, making the rest deeper, and "consecutive positive induction" - increase in excitability that replaces the inhibition processes, and results in easier work renewal. This phenomenon underlies Sechenov's ideas about "active rest" and the possibility to modulate the processes of fatigue development.

Our data show that cognitive task, at least the one that includes motor component, can improve concurrent motor work performance and reduce muscle fatigue development. This possible primarily motor effect should be taken into account by researchers of higher cognitive functions, especially when motor response (e. g. widely used button press) serves as the only measure of cognitive performance. There was also a tendency for data from active discrimination task without motor component to occupy the intermediate position between data from passive task and active discrimination with motor component. Our results confirm the idea that the process of fatigue development in course of motor work execution, as well as cognitive performance, can be modulated, and, possibly, even controlled.

Optimal combination of cognitive and physical activities may increase work capacity and performance and accelerate recuperation of fatigue after work (7). Results obtained in the present study, in part, support the idea that "diverting activity"mental activity may influence muscle fatigue development and recuperation.

Work was made with financial support of the Federal Target Program "Scientific and educational personnel of innovative Russia" GC 14.740.11.0232.

\section{References}

[1] Bigland-Ritchie, B., Rice, C. L., Garland, S. J., Walsh, M. L. (1995) Task-dependent factors in fatigue of human voluntary contractions. In Fatigue, ed. Gandevia, S. C., Enoka, R. M., McComas, A. J., Stuart, D. G., Thomas, C. K. 1995, 361-380.

[2] Enoka RM, Stuart DG (1992) Neurobiology of muscle fatigue. J Appl Physiol 72, 1631-1648

[3] .Mathiassen, S. E. (2006). "Diversity and variation in biomechanical exposure: what is it, and why would we like to know?" Appl Ergon 37(4): 419-427

[4] Decker L.M., Myers S.A., Rodrigues-Aranda S.E., Potter J.F., Stergiou N. 2009. Performance of dual-task requiring language perception, attention, and executive control processes have differential effects on stride width sin young adults. Program No. 662.18. 2009 Neuroscience Meeting Planner. Chicago, IL: Society for Neuroscience, 2009. Online

[5] Davranche, K., Pichon, A., 2005. Critical flicker frequency threshold increment after an exhausting exercise. Journal of Sport \& Exercise Psychology 27, 515-520

[6] . Audiffren, M., Tomporowski, P., Zagrodnik, J., 2008. Acute aerobic exercise and information processing: energizing motor processes doing a choice reaction time task. Acta Psychologica 129, 410-419

[7] Asmussen, E. and B. Mazin (1978). "Recuperation after muscular fatigue by "diverting activities"." Eur J Appl Physiol Occup Physiol 38(1): 1-7 